\title{
Eriodictyol, a plant flavonoid, attenuates LPS-induced acute lung injury through its antioxidative and anti-inflammatory activity
}

\author{
GUANG-FA ZHU ${ }^{1}$, HONG-JUAN GUO ${ }^{2}$, YAN HUANG ${ }^{2}$, CHUN-TING WU W $^{2}$ and XIANG-FENG ZHANG ${ }^{2}$ \\ ${ }^{1}$ Departments of Infectious Diseases and ${ }^{2}$ Respiratory and Critical Care Medicine, Beijing Anzhen Hospital, \\ Capital Medical University, Beijing Institute of Heart, Lung and Blood Vessel Diseases, Beijing 100029, P.R. China
}

Received September 2, 2014; Accepted May 15, 2015

DOI: $10.3892 /$ etm.2015.2827

\begin{abstract}
Acute lung injury (ALI) is characterized by excessive inflammatory responses and oxidative injury in the lung tissue. It has been suggested that anti-inflammatory or antioxidative agents could have therapeutic effects in ALI, and eriodictyol has been reported to exhibit antioxidative and anti-inflammatory activity in vitro. The aim of the present study was to investigate the effect of eriodictyol on lipopolysaccharide (LPS)-induced ALI in a mouse model. The mice were divided into four groups: Phosphate-buffered saline-treated healthy control, LPS-induced ALI, vehicle-treated ALI (LPS + vehicle) and eriodictyol-treated ALI (LPS + eriodictyol). Eriodictyol $(30 \mathrm{mg} / \mathrm{kg}$ ) was administered orally once, 2 days before the induction of ALI. The data showed that eriodictyol pretreatment attenuated LPS-induced ALI through its antioxidative and anti-inflammatory activity. Furthermore, the eriodictyol pretreatment activated the nuclear factor erythroid-2-related factor 2 (Nrf2) pathway in the ALI mouse model, which attenuated the oxidative injury and inhibited the inflammatory cytokine expression in macrophages. In combination, the results of the present study demonstrated that eriodictyol could alleviate the LPS-induced lung injury in mice by regulating the Nrf2 pathway and inhibiting the expression of inflammatory cytokines in macrophages, suggesting that eriodictyol could be used as a potential drug for the treatment of LPS-induced lung injury.
\end{abstract}

\section{Introduction}

Acute lung injury (ALI) and acute respiratory distress syndrome (ARDS), its more severe form, are acute respiratory failure syndromes, which are characterized by severe

Correspondence to: Dr Guang-Fa Zhu, Department of Infectious Diseases, Beijing Anzhen Hospital, Capital Medical University, Beijing Institute of Heart, Lung and Blood Vessel Diseases, 2 Anzhen Road, Beijing 100029, P.R. China

E-mail: guangfazhu_2014@126.com

Key words: eriodictyol, lipopolysaccharide-induced acute lung injury, acute lung injury, nuclear factor erythroid-2-related factor 2 pathway hypoxemia, pulmonary edema and neutrophil accumulation in the lungs (1). The occurrence of ALI/ARDS may be a result of critical illnesses of diverse etiologies; these illnesses may be a result of direct injury to the lung, such as toxic inhalation, aspiration, pneumonia and near-drowning, or occur through indirect causes, e.g. sepsis, pancreatitis, burns, gynecological conditions (amniotic fluid embolism, eclampsia, placental abruption) and massive blood transfusion. The clinical incidence of ALI/ARDS is high and the condition is associated with marked mortality and morbidity $(2,3)$.

Inflammation is an early response of the vascular tissues to infection, injuries and harmful stimuli, such as pathogens and irritants; it is involved in non-specific immune responses for neutralizing invaders and repairing damaged cells and it initiates the healing processes (4). Lipopolysaccharide (LPS) is a structural component of the outer membrane of Gram-negative bacteria and is known to induce inflammation (5). LPS binds to and signals through the Toll-like receptor 4 , leading to a rapid release of pro-inflammatory cytokines, such as tumor necrosis factor (TNF). TNF subsequently acts through its membrane receptor 1 complex I (6-8) to activate multiple downstream effectors, such as the transcription factor nuclear factor $\kappa \mathrm{B}(\mathrm{NF}-\kappa \mathrm{B})$, to further induce the production of pro-inflammatory cytokines, including interleukin (IL)-6, IL-1 $\beta$ and macrophage inflammatory protein 2 (MIP-2), thereby amplifying the inflammatory response $(9,10)$. Excessive inflammatory reactions, particularly the activation of neutrophils and macrophages, have been implicated in the pathogenesis of ARDS $(11,12)$. In addition, increased levels of oxidants, if unchecked, can become deleterious to the cells, since they are responsible for oxidative stress (acute or chronic), which can cause tissue damage and apoptotic cell death. Oxidative stress plays a key role in the pathogenesis of an extensive range of diseases, including Alzheimer's and Parkinson's diseases (13), cardiovascular and pulmonary disorders $(14,15)$ and, in particular, ALI and primary and secondary infections.

Inflammation and oxidative stress play a critical role in the pathogenic process, which is generated by the innate immune response to LPS-induced acute tissue injury. Furthermore, inflammation and oxidative stress are inextricably linked, as each generates and reinforces the other; for example, through the activation of $\mathrm{NF}-\kappa \mathrm{B}$, oxidative stress promotes the recruitment and activation of resident cells and leukocytes, thereby 
evoking inflammation (16), while activated resident cells, leukocytes and macrophages induce oxidative stress through the generation of reactive oxygen, chlorine and nitrogen species.

The transcription factor nuclear factor erythroid-2-related factor 2 (Nrf2) is a basic leucine zipper transcription factor and a member of the cap ' $n$ ' collar transcription factor family (17). Nrf2 is responsible for both the constitutive and the inducible expression of antioxidant response element-regulated genes. Nrf2 activation is an effective antioxidant defensive mechanism that is used by host cells against oxidative stress. Nrf2 has been shown to be the master regulator of several hundreds of genes that are involved in the antioxidant defense response (18).

Eriodictyol, a flavonoid isolated from the Chinese herb Dracocephalum rupestre, has long been established as an antioxidant and anti-inflammatory agent. Notably, eriodictyol is distributed in common foods and has been identified to have beneficial biological activities $(19,20)$. Eriodictyol has been found to suppress nitric oxide production, NF- $\mathrm{KB}$ activation and mitogen-activated protein kinase phosphorylation in mouse macrophages (21). Based on these previously observed properties of eriodictyol, the aim of the present study was to use a mouse model to investigate whether eriodictyol could exert a protective effect in LPS-induced ALI.

\section{Materials and methods}

Mice. Specific pathogen-free female C57BL/6 mice (8-10 weeks old) were purchased from the SLRC Laboratory Animal Co., Ltd. (Shanghai, China). The animals were maintained under barrier conditions and kept at $22-25^{\circ} \mathrm{C}$ under a 12 -h light/dark cycle. The mice were randomly divided into four groups: Phosphate buffered saline (PBS)-treated healthy control $(n=10)$, LPS-induced ALI ( $\mathrm{n}=10)$, vehicle-treated ALI (LPS + vehicle, $\mathrm{n}=10$ ) and eriodictyol-treated ALI (LPS + eriodictyol, $\mathrm{n}=10)$. All experimental procedures were carried out in strict accordance with the NIH Guidelines for the Care and Use of Laboratory Animals, and animal handling was performed following the dictates of the National Animal Welfare Law of China.

Establishment of the ALI mouse model. Eighty female C57BL/6 mice were anesthetized by an intraperitoneal injection of $150 \mathrm{mg} / \mathrm{kg}$ ketamine $\mathrm{HCl}$ and $65 \mu \mathrm{g} / \mathrm{kg}$ xylazine hydrochloride. E. coli LPS (Sigma-Aldrich, St. Louis, MO, USA) was instilled intratracheally ( $25 \mu \mathrm{g}$ in $50 \mu \mathrm{l}$ sterile saline) during inspiration. The control mice received PBS instillation, while the eriodictyol- and vehicle-treated mice received eriodictyol $(30 \mathrm{mg} / \mathrm{kg}$, dissolved in PBS) and vehicle (PBS), respectively, orally 2 days prior to the induction of ALI. The mice were then sacrificed by an intravenous injection of thiopental $24 \mathrm{~h}$ after the induction of ALI. The thorax was opened and the blood was sampled by cardiac puncture. Simultaneously, three bronchoalveolar lavage (BAL) procedures were performed, each using $0.5 \mathrm{ml}$ normal saline. The blood was centrifuged $\left(2,000 \mathrm{xg}\right.$, for $10 \mathrm{~min}$ at $\left.4^{\circ} \mathrm{C}\right)$ and the serum was stored for further processing; the survival curve was then depicted using the Kaplan-Meier method.

BAL fluid (BALF) collection and cell counting. Three simultaneous BAL procedures were carried out using $0.5 \mathrm{ml}$ sterile saline, and the BALF was collected. The recovered lavage was centrifuged at $3,000 \times \mathrm{g}$ for $10 \mathrm{~min}$ at $4^{\circ} \mathrm{C}$. The cell-free supernatants that were obtained from the first procedure were stored at $-20^{\circ} \mathrm{C}$ for further analysis for inflammatory cytokine and protein concentration by ELISA. The BALF cell pellet was resuspended in PBS for cell counting.

Morphological assessment of lung injury. Mice were anesthetized by an intraperitoneal injection of $100 \mathrm{mg} / \mathrm{kg}$ ketamine mixed with $10 \mathrm{mg} / \mathrm{kg}$ xylazine. Following exposure, the lung tissues were perfused with PBS and fixed by an injection of $10 \%$ formalin into the trachea. Following tracheal ligation, the lung tissues were incubated in $4 \%$ formalin overnight at $4{ }^{\circ} \mathrm{C}$. The lung tissues were then embedded with paraffin, sectioned at 5- $\mu \mathrm{m}$ thickness and stained with hematoxylin and eosin.

For the determination of the lung injury score, the histological images were evaluated by an assessor who was initially blinded to the study groups. Lung injury was scored based on edema, neutrophil inflitration, hemorrhage, bronchiole epithelial desquamation and hyaline membrane formation (22). The score ranged between 0 and 4, based on severity: 0 , indicated no injury; 1 , indicated modest injury (including limited congestion and interstitial edema, but no interstitial neutrophilic infiltrate with inflammatory cells in the alveolar spaces); 2, indicated intermediate injury (including mild congestion, interstitial edema, and interstitial neutrophilic infiltrate with inflammatory cells in the alveolar spaces); 3 , indicated widespread injury (including more prominent congestion and interstitial edema with neutrophils partially filling the alevolar spaces); and 4, indicated widespread injury (including most prominent, marked congestion, and interstitial edema with neutrophilic infiltrate nearly filling the alveolar spaces or with pulmonary consolidation). The average values were considered a semi-quantitative histological index of lung injury.

Measurement of lung myeloperoxidase (MPO) activity. The frozen right lower lobe lung samples were weighed and homogenized in $2 \mathrm{ml}$ of $50 \mathrm{mmol} / \mathrm{l}$ phosphate buffer at $\mathrm{pH} 6.0$ containing $0.5 \%$ hexadecyltrimethylammonium bromide (Sigma-Aldrich). The samples were then frozen on dry ice and thawed at room temperature thrice, following which they were sonicated. The suspensions were subsequently centrifuged at 2,000 $\mathrm{xg}$ for $15 \mathrm{~min}$ at $4^{\circ} \mathrm{C}$. The MPO activity in the supernatant was assessed by measuring the $\mathrm{H}_{2} \mathrm{O}_{2}$-dependent oxidation of $o$-dianisidine dihydrochloride (Sigma-Aldrich), as previously described (23). A total of $0.1 \mathrm{ml}$ supernatant was mixed with $2.9 \mathrm{ml}$ phosphate buffer $(50 \mathrm{mmol} / \mathrm{l}, \mathrm{pH} 6.0)$ containing $0.0005 \% \mathrm{H}_{2} \mathrm{O}_{2}$ (Sigma-Aldrich) and $0.167 \mathrm{mg} / \mathrm{ml}$ $o$-dianisidine dihydrochloride (Sigma-Aldrich). The change in absorbance was measured at $460 \mathrm{~nm}$ using a spectrophotometer. The MPO activity was expressed as the change in absorbance per minute and was further normalized to the total protein content of the supernatant.

Measurement of lung tissue wet/dry ratio. The left lungs were extracted and the wet weights were obtained. The lung tissues were then reweighed 3 days after drying at $80^{\circ} \mathrm{C}$. The wet/dry ratio was calculated as follows: wet/dry ratio $=($ wet weight - dry weight)/dry weight (24). 
Lactate dehydrogenase ( $L D H$ ) assay. The LDH activity in the BALF was measured using a Sigma LDH determination kit (Sigma-Aldrich). Briefly, $200 \mu \mathrm{l}$ BALF supernatant was added to $2.5 \mathrm{ml}$ reagent $\mathrm{A}$ for $1 \mathrm{~min}$, prior to $100 \mu \mathrm{l}$ reagent $\mathrm{B}$ being added. The absorbance at $340 \mathrm{~nm}$ was recorded every minute for $3 \mathrm{~min}$ for the purpose of confirming the linearity of the reaction. The LDH activity was expressed in U/l. Using LDH standard (Sigma-Aldrich) it was confirmed that there was a linear increase in absorbance with increasing LDH concentrations.

Protein concentration in the BALF. BAL was performed as described in a previous study (25). Briefly, following sacrifice, the trachea was exposed and intubated using a tracheal cannula. BAL was performed three times by repeatedly flushing the airways and lungs with $0.5 \mathrm{ml}$ cold saline. The pooled BALF was collected on ice and centrifuged at $500 \mathrm{x}$ g for $5 \mathrm{~min}$ at $4{ }^{\circ} \mathrm{C}$. The supernatant was then stored at $-20^{\circ} \mathrm{C}$ for further analysis. The determination of protein concentrations in the cell-free BALF was performed using Bio-Rad protein assay reagents (Bio-Rad, Hercules, CA, USA). In the same fashion, a standard curve was generated using bovine serum albumin.

ELISA. The TNF- $\alpha$, IL-6, IL-1 $\alpha$ and MIP-2 concentrations were measured by ELISA according to the manufacturer's instructions. The experiment was repeated thrice and the results were presented as the mean value of the three experiments.

Survival study of mice with LPS-induced ALI. The possibility that pretreatment with eriodictyol could confer protection against LPS-induced ALI was assessed. The mice were randomly divided into four experimental groups $(n=10$ per group), as mentioned previously, and the survival rates were recorded at $120 \mathrm{~h}$.

Measurement of oxidative stress in vivo. The lung tissues were homogenized in $0.01 \mathrm{M} \mathrm{PBS}$ ( $\mathrm{pH} 7.4$ ) and clarified by centrifugation at $10,000 \mathrm{xg}$ for $10 \mathrm{~min}$ at $4^{\circ} \mathrm{C}$. The levels of $\mathrm{H}_{2} \mathrm{O}_{2},{ }^{\circ} \mathrm{OH}$ and malondialdehyde (MDA) were subsequently measured in order to determine the level of oxidative stress in the lungs. The assays were performed in accordance with the instructions of the manufacturers of the $\mathrm{H}_{2} \mathrm{O}_{2}$ (Abcam, Cambridge, MA, USA), ${ }^{\circ} \mathrm{OH}$ (Nanjing Jiancheng Bioengineering Research Institute, Nanjing, China) and MDA kits (Abcam). The $\mathrm{H}_{2} \mathrm{O}_{2}$, 'OH and MDA levels corresponded to the level of oxidative stress in vivo.

Western blot analysis. The tissues and macrophages were immediately homogenized and the proteins were extracted according to the instructions of the total protein extraction kit (G-Biosciences, St. Louis, MO, USA). The protein concentration was determined using the bicinchoninic acid protein assay kit (Sigma-Aldrich). The protein samples were then subjected to 10\% SDS-PAGE and transferred to a polyvinylidene difluoride membrane. Following blocking with 5\% milk, the membrane was incubated with the indicated primary antibodies: Rabbit polyclonal anti-Nrf2 (1:200; cat. no. sc-722; Santa Cruz Biotechnology, Inc., Dallas, TX, USA), mouse monoclonal anti-thioredoxin 1 (Trx1) (1:200; cat. no. sc-13526; Santa Cruz
Biotechnology, Inc.) and rabbit polyclonal anti- $\beta$-actin (1:1,000; cat. no. A2066; Sigma-Aldrich) at room temperature for $2 \mathrm{~h}$. The membranes were then incubated with goat anti-rabbit $(1: 1,000$; cat. no. A0545; Sigma-Aldrich) and goat anti-mouse (1:1,000; cat. no. HAF007, R\&D Systems, Inc., Minneapolis, MN, USA) secondary antibodies at room temperature for $1 \mathrm{~h}$, and the protein bands were visualized by the enhanced chemiluminescence system (Pierce Biotechnology, Inc., Rockford, IL, USA). Blots were analyzed using ImageJ $1.48 \mathrm{~V}$ software (National Institutes of Health, Bethesda, MD, USA).

Reverse transcription quantitative polymerase chain reaction $(R T-q P C R)$. The total RNA was isolated from macrophages from differentially treated ALI mice using TRIzol ${ }^{\circledR}$ reagent (Invitrogen Life Technologies, Carlsbad, CA, USA), and RT-qPCR was performed as previously reported (Life Technologies, Carlsbad, CA, USA) (26). PCR was conducted using a Plexor ${ }^{\circledR}$ qPCR and qRT-PCR system (Promega Corporation, Madison, WI, USA) on an ABI 7900 Fast thermal cycler (Applied Biosystems Life Technologies, Foster City, CA, USA). The following primer pairs were used in the analysis: Mouse hypoxanthine phosphoribosyltransferase, 5'-CTGGTGAAAAGGACCTCTCG-3' (forward) and 5'-TGAAGTACTCATTATAGTCAAGGGCA-3' (reverse); mouse Nrf2, 5'-TTGGCAGAGACATTCCCATTTG-3' (forward) and 5'-AAACTTGCTCCATGTCCTGCTCTA-3' (reverse); mouse Trx1, 5'-TGCTACGTGGTGTGGACC TTGC-3' (forward) and 5'-ACCGGAGAACTCCCCCAC CT-3' (reverse); mouse IL-6, 5'-CCAGAAACCGCTATG AAGTTCC-3' (forward) and 5'-TCACCAGCATCAGTCCCA AG-3' (reverse); mouse TNF- $\alpha, 5$ '-CTCCAGGCGGTGCCT ATGT-3' (forward) and 5'-GAAGAGCGTGGTGGC CC-3'(reverse); mouse MIP-2, 5'-TCCAGAGCTTGAGTG TGACG-3' (forward) and 5'- TCAGGTACGATCCAGGCT TC-3' (reverse); mouse IL-1 $\beta, 5^{\prime}$-CAACCAACAAGTGAT ATTCTCCATG-3' (forward) and 5'-GATCCACACTCTCCA GCTGCA-3' (reverse) (Sangon Biotech Co., Ltd., Shanghai, China). The PCR cycling conditions were as follows: $95^{\circ} \mathrm{C}$ for $10 \mathrm{~min}$, followed by 40 cycles at $95^{\circ} \mathrm{C}$ for $15 \mathrm{sec}$ and $60^{\circ} \mathrm{C}$ for $60 \mathrm{sec}$, a final extension step at $95^{\circ} \mathrm{C}$ for $60 \mathrm{sec}$, and a dissociation curve analysis. Relative quantification (RQ) was determined using the following formula: $R Q=2^{-\Delta C t}\left(\Delta \mathrm{Ct}=\mathrm{Ct}_{\text {gene }}\right.$ of interest $-\mathrm{Ct}_{\text {endogenous control }}$ ).

Macrophage culture. Bone marrow cells from mouse femur or tibia were harvested and selected using RPMI-1640 medium as previously described (27). A week later, bone marrow-derived macrophages were replated, and untreated macrophages were stimulated with LPS $(100 \mathrm{ng} / \mathrm{ml})$ for $24 \mathrm{~h}$ or LPS and eriodictyol or vehicle for $24 \mathrm{~h}$. The cells and culture supernatant were then collected in order to determine the inflammatory cytokine expression.

Statistical analysis. All data were analyzed using SPSS 13.0 (SPSS Inc., Chicago, IL, USA) software and expressed as the mean \pm standard error of the mean. One-way analysis of variance followed by Fisher's protected least significant difference test were used to assess significant differences, and $\mathrm{P}<0.05$ was considered to indicate a statistically significant difference. 

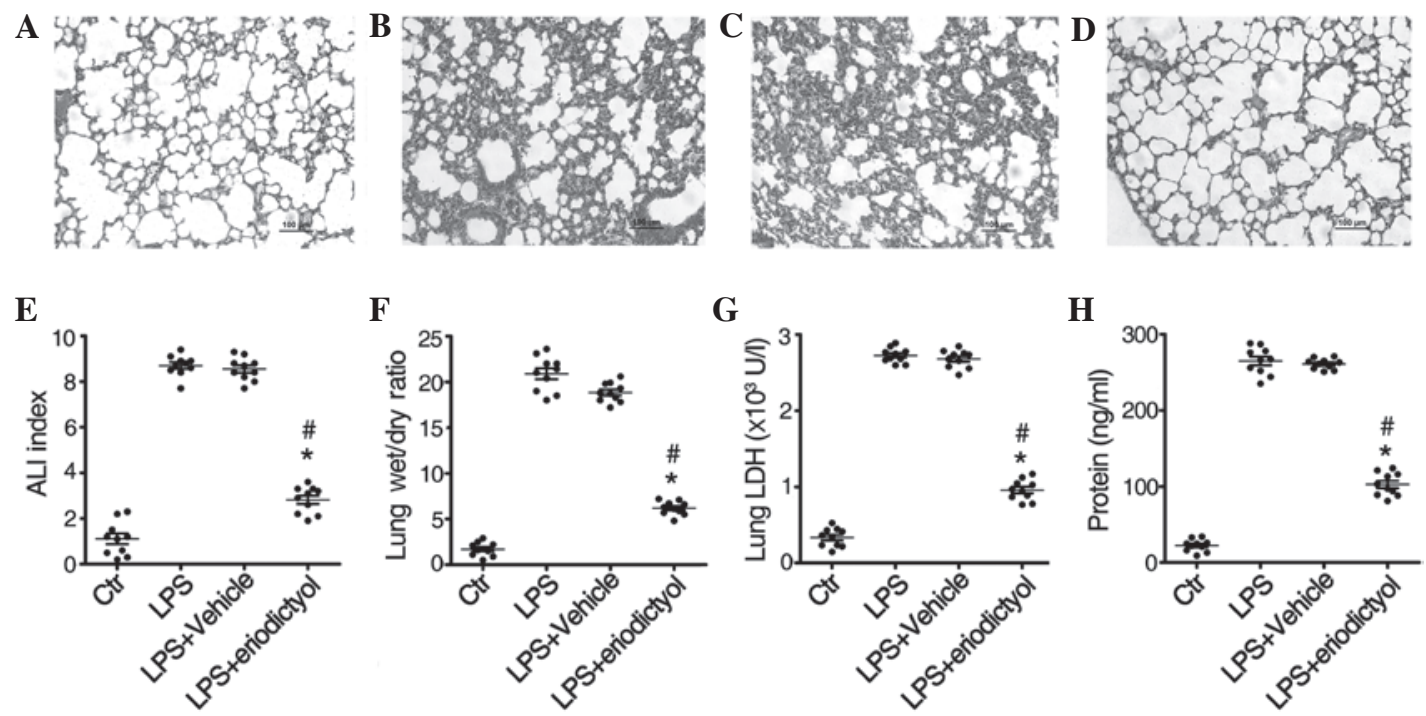

Figure 1. Morphological alterations in the lungs as determined by photomicrography. (A-D) Photomicrograph of a lung section from the (A) phosphate-buffered saline-treated healthy control group, (B) LPS-induced ALI group, (C) vehicle-treated ALI group and (D) eriodictyol-treated ALI group (hematoxylin \& eosin staining; magnification, x200). (E) Lung injury index, (F) lung wet/dry ratio, (G) lung tissue LDH level and (H) protein concentration in the bronchoalveolar lavage fluid of differentially treated ALI mice. Data are presented as the mean \pm standard error $(n=10)$. " $\mathrm{P}<0.05$ compared with the LPS-induced ALI group; ${ }^{\text {"}} \mathrm{P}<0.05$ compared with the vehicle-treated ALI group. ALI, acute lung injury; Ctr, control; LPS, lipopolysaccharide; LDH, lactate dehydrogenase.
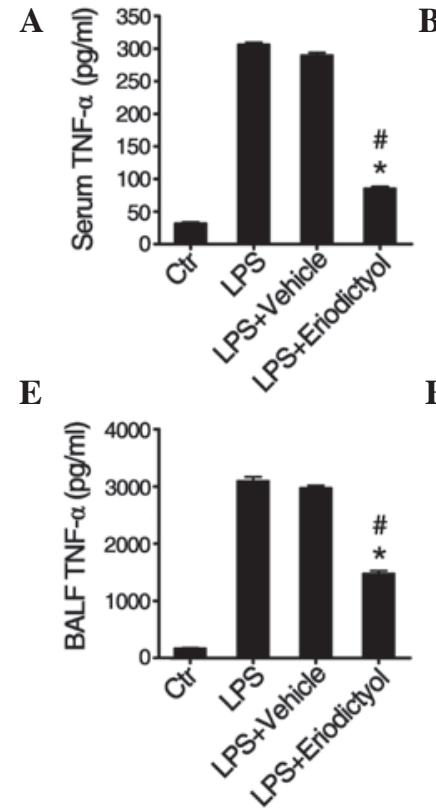

B

$\mathbf{F}$
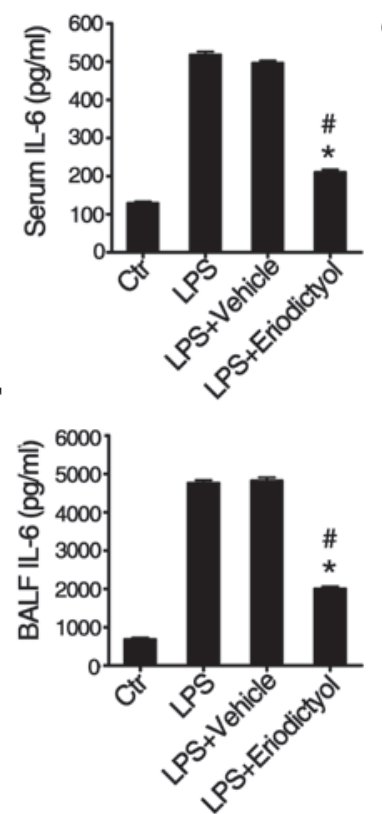

C

G
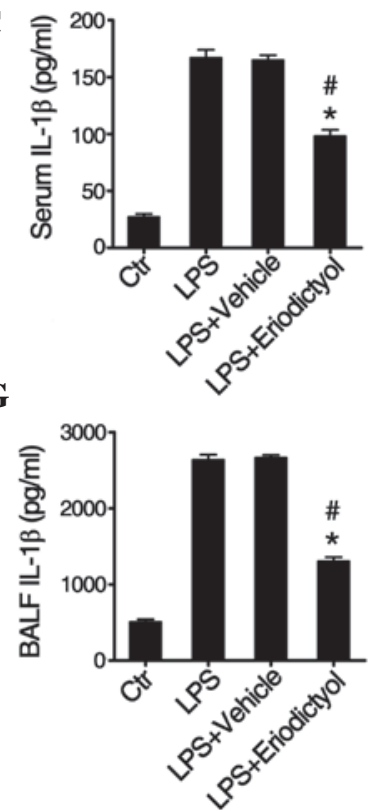

D
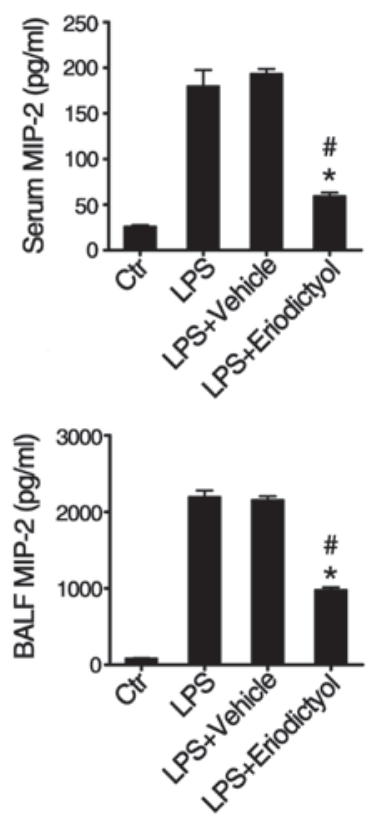

Figure 2. Effect of eriodictyol on LPS-induced pro-inflammatory cytokine secretion in the serum and BALF of differentially treated ALI mice. (A-D) Levels of (A) TNF- $\alpha$, (B) IL-6, (C) IL-1 $\beta$ and (D) MIP-2 in the serum and (E-H) levels of (E) TNF- $\alpha$, (F) IL-6, (G) IL-1 $\beta$ and (H) MIP-2 in the BALF of differentially treated ALI mice were measured using ELISA. Data are presented as the mean \pm standard error $(n=10)$. ${ }^{*} \mathrm{P}<0.05$ compared with the LPS-induced ALI group; ${ }^{\#} \mathrm{P}<0.05$ compared with the vehicle-treated ALI group. ALI, acute lung injury; Ctr, control; LPS, lipopolysaccharide; MIP-2, macrophage inflammatory protein 2; TNF- $\alpha$, tumor necrosis factor $\alpha$; IL, interleukin, BALF, bronchoalveolar lavage fluid.

\section{Results}

Effect of eriodictyol pretreatment on ALI. The LPS-induced ALI animal model is a common experimental model used for the investigation of molecular mechanisms and drug efficacy in ALI (28). To observe the effect of eriodictyol on ALI, an LPS-induced ALI animal model was therefore established. As shown in Fig. 1, the morphological examination following LPS induction revealed that several histopathological alterations, including cell structure destruction, neutrophil infiltration, alveolar wall thickening and lung edema, had occurred in the lung tissue (Fig. 1B), as compared with the PBS-treated control group (Fig. 1A). When compared with the vehicle-treated ALI mice (Fig. 1C), however, the LPS-induced pathological symptoms were markedly improved following pretreatment with eriodictyol (Fig. 1D), suggesting that eriodictyol has a protective effect against LPS-induced ALI. 
In order to further evaluate the protective effect of eriodictyol in ALI, the lung injury index, lung tissue wet/dry ratio, $\mathrm{LDH}$ and protein concentration in the lung BALF of differentially treated ALI mice were determined. It was found that, compared with the PBS-treated healthy control group, eriodictyol pretreatment decreased the lung injury index (Fig. 1E), lung wet/dry ratio (Fig. 1F), LDH level (Fig. 1G) and BALF protein concentration (Fig. $1 \mathrm{H}$ ), which had been elevated due to LPS stimulation. These results were in agreement with the effect of eriodictyol on lung tissue histology.

Eriodictyol pretreatment decreases the production of inflammatory cytokines in LPS-induced ALI mice. Pro-inflammatory cytokines, including TNF- $\alpha$, IL-6, IL-1 $\beta$ and MIP-2, play a critical role in the pathogenesis of ALI, and therefore the effect of eriodictyol on TNF- $\alpha$, IL-6, IL-1 $\beta$ and MIP-2 production in the serum and BALF of differentially treated ALI mice was measured by ELISA $24 \mathrm{~h}$ after LPS induction. As shown in Fig. 2, LPS caused a significant increase in the serum levels of TNF- $\alpha$ (Fig. 2A), IL-6 (Fig. 2B), IL-1 $\beta$ (Fig. 2C) and MIP-2 (Fig. 2D), as well as in the corresponding lung BALF levels (Fig. 2E-H), of the differentially treated ALI mice; however, the LPS-induced increase in inflammatory cytokines observed in the serum and lung BALF of the vehicle-pretreated ALI mice was reversed by the eriodictyol pretreatment (Fig. 2). These results demonstrated that eriodictyol could inhibit the inflammatory response in LPS-induced ALI.

Inhibition of MPO activity and inflammatory neutrophil accumulation in the lung tissues by eriodictyol pretreatment. LPS-induced ALI is characterized by an increase in neutrophils and MPO activity in the lung tissues $(29,30)$. Furthermore, an increased MPO activity reflects polymorphonuclear neutrophil accumulation in the lungs. For that reason, the MPO activity in the lung tissue homogenates and the number of neutrophils in the lung BALF of differentially treated ALI mice were examined. Following LPS induction, a significant increase was observed in the MPO activity (Fig. 3A) and the number of neutrophils (Fig. 3B) in the lung tissues, compared with the PBS-treated healthy control group; however, this increase in MPO activity (Fig. 3A) and neutrophils (Fig. 3B) was eliminated by the eriodictyol pretreatment, as compared with the LPS-induced ALI or vehicle-treated groups.

Improved survival rate of LPS-induced ALI mice following eriodictyol pretreatment. The survival rate, which was strongly indicative of the protective effect of eriodictyol on LPS-induced ALI, was examined in differentially treated ALI mice as previously described (31). It was found that, compared with the LPS-induced or vehicle-treated ALI groups, the survival rate of the LPS-induced ALI mice was significantly improved following eriodictyol administration (Fig. 4).

Decrease in oxidative stress levels in LPS-induced ALI mice following eriodictyol pretreatment. Considerable experimental evidence supports the role of oxidants, including $\mathrm{H}_{2} \mathrm{O}_{2}$, - $\mathrm{OH}$ and MDA, and oxidative injury in the pathogenesis of ALI/ARDS $(32,33)$; therefore, the levels of $\mathrm{H}_{2} \mathrm{O}_{2},{ }^{\circ} \mathrm{OH}$ and MDA in the lung tissues from differentially treated ALI mice were determined. The results of the in vivo assays revealed
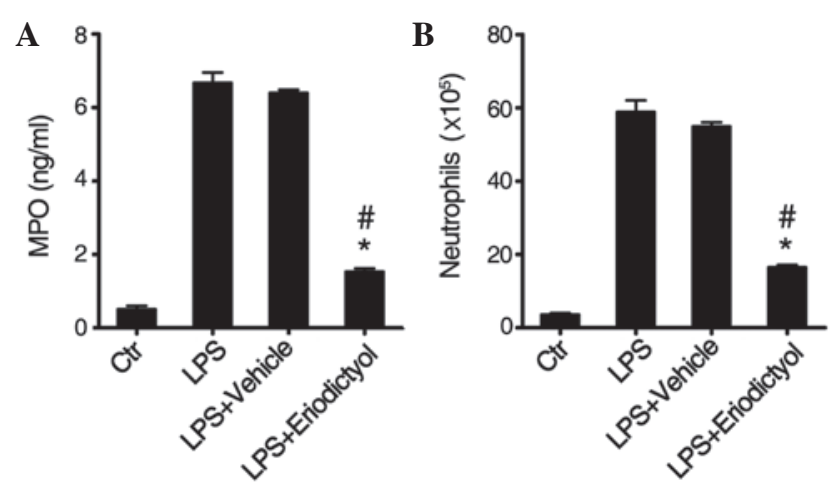

Figure 3. Effects of eriodictyol on MPO activity and neutrophil accumulation in lung tissues. (A) MPO activity in the lung tissue homogenates of differentially treated ALI mice. (B) Neutrophil count in the lung bronchoalveolar lavage fluid of differentially treated ALI mice. Data are presented as the mean \pm standard error $(n=10)$. ${ }^{*} \mathrm{P}<0.05$ compared with the LPS-induced ALI group; ${ }^{\#} \mathrm{P}<0.05$ compared with the vehicle-treated ALI mice. ALI, acute lung injury; Ctr, control; LPS, lipopolysaccharide; MPO, myeloperoxidase.

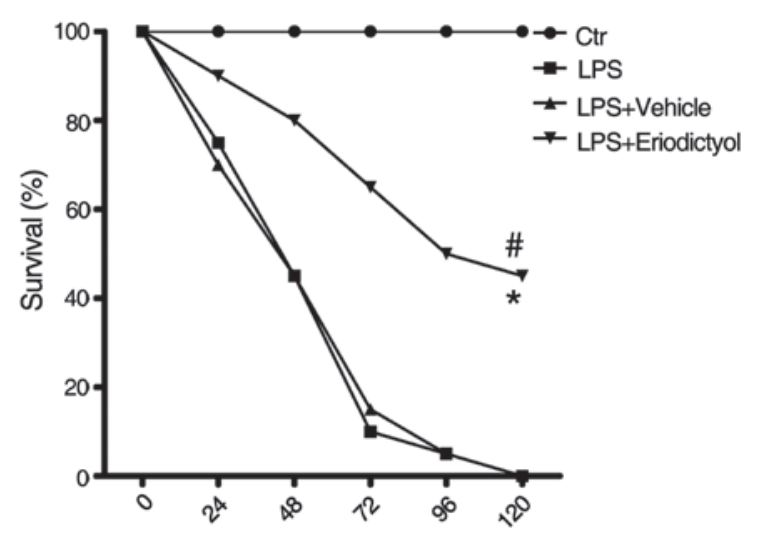

Figure 4. Effect of eriodictyol on LPS-induced mortality in differentially treated ALI mice. The mice were challenged with LPS, with or without eriodictyol pretreatment, and the survival rates were observed after 0, 24, 48, 72, 96 and $120 \mathrm{~h}$. Eriodictyol pretreatment prevented LPS-induced mortality. ${ }^{*} \mathrm{P}<0.05$ compared with the LPS-induced ALI group; ${ }^{\#} \mathrm{P}<0.05$ compared with the vehicle-treated ALI group. ALI, acute lung injury; Ctr, control; LPS, lipopolysaccharide.

increased levels of $\mathrm{H}_{2} \mathrm{O}_{2}$ (Fig. 5A), ${ }^{\circ} \mathrm{OH}$ (Fig. 5B) and MDA (Fig. 5C) in the lung tissue homogenates from LPS-induced mice, compared with those from the control group; however the eriodictyol pretreatment decreased the accumulation of $\mathrm{H}_{2} \mathrm{O}_{2}$ (Fig. 5A), ${ }^{\circ} \mathrm{OH}$ (Fig. 5B) and MDA (Fig. 5C) in the lung tissues. In combination, these results indicated that eriodictyol could attenuate LPS-induced ALI through its antioxidative activity.

Eriodictyol pretreatment enhances Trxl expression by regulating the $\mathrm{Nrf} 2$ pathway. A previous study reported that eriodictyol could activate the Nrf2 pathway and enhance the expression of Trx1 in vitro (34), while a different study showed that Nrf2 could regulate a series of antioxidant-responsive genes (35). Trx1 is a small, ubiquitous protein that acts as a redox protein in response to various oxidative stress conditions (36). As shown in Fig. 6, treatment with eriodictyol significantly increased the mRNA expression of Nrf2 (Fig. 6A) and Trx1 (Fig. 6B) in the eriodictyol-treated ALI 

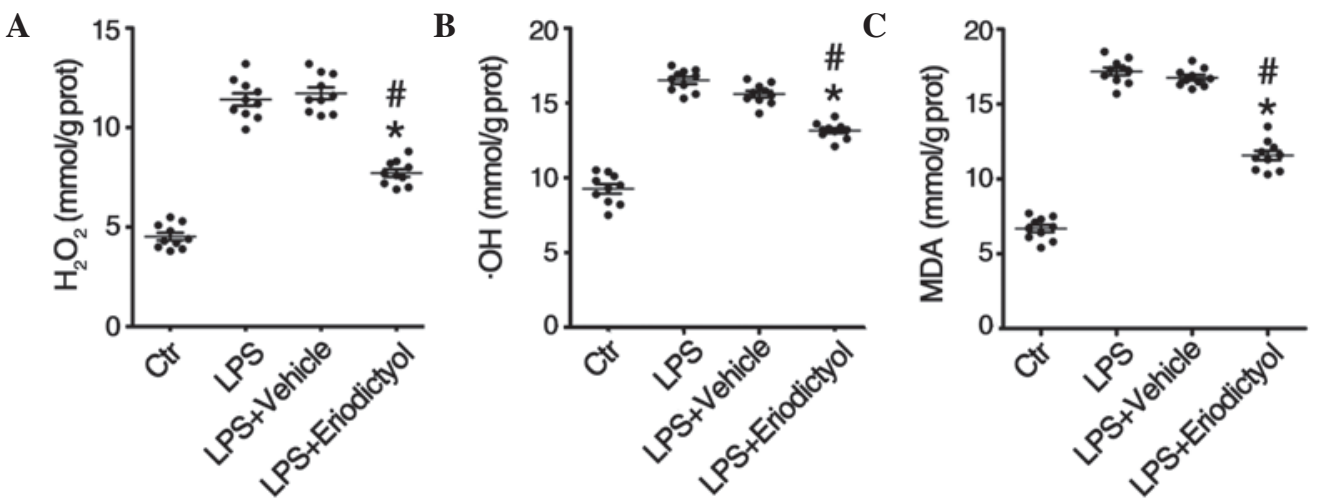

Figure 5. (A) $\mathrm{H}_{2} \mathrm{O}_{2}$, (B) ${ }^{\circ} \mathrm{OH}$ and (C) MDA levels in the homogenized lung tissue. Eriodictyol decreased the oxidative stress-related production of these compounds, which had been increased by LPS. Data are presented as the mean \pm standard error $(n=10)$. ${ }^{*} \mathrm{P}<0.05$ compared with the LPS-induced ALI group; ${ }^{\#} \mathrm{P}<0.05$ compared with the vehicle-treated ALI group. ALI, acute lung injury; Ctr, control; LPS, lipopolysaccharide; MDA, malondialdehyde.
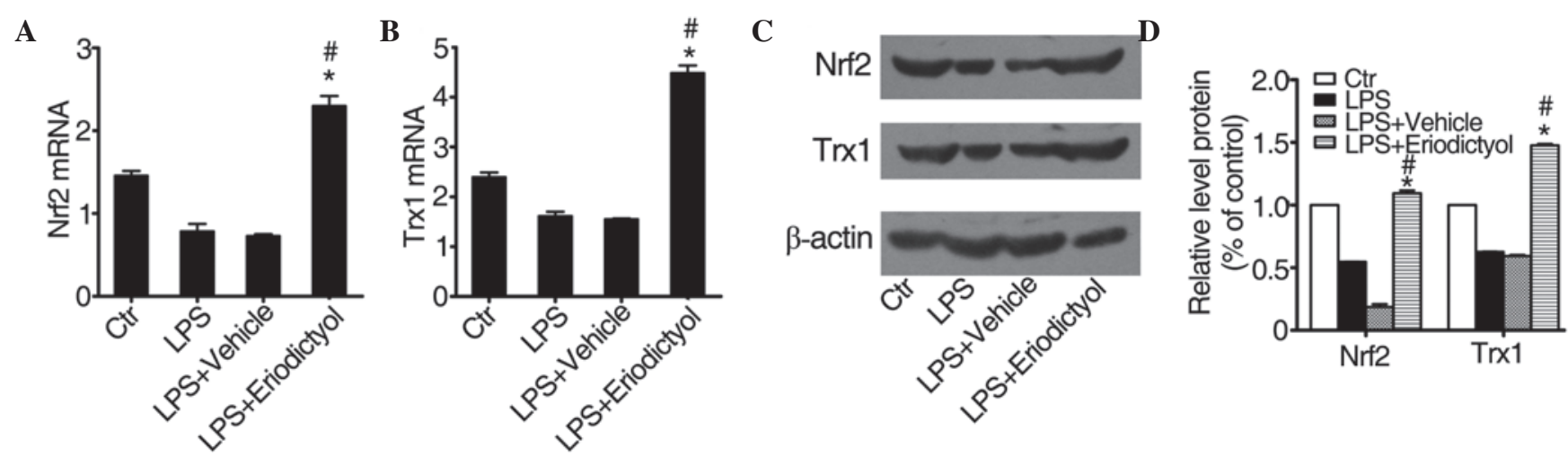

Figure 6. Effect of eriodictyol on Nrf2 and Trx1 expression in lung tissues. The (A and B) reverse transcription-quantitative polymerase chain reaction and (C and D) western blot analysis results showed that eriodictyol upregulated Nrf2 and Trx1 expression. Data are presented as the mean \pm standard error ( $\mathrm{n}=10$ ). ${ }^{*} \mathrm{P}<0.05$ compared with the LPS-induced ALI group; ${ }^{\mathrm{P}} \mathrm{P}<0.05$ compared with the vehicle-treated ALI group. ALI, acute lung injury; Ctr, control; LPS, lipopolysaccharide; Nrf2, nuclear factor erythroid-2-related factor 2; Trx1, thioredoxin isoform 1.
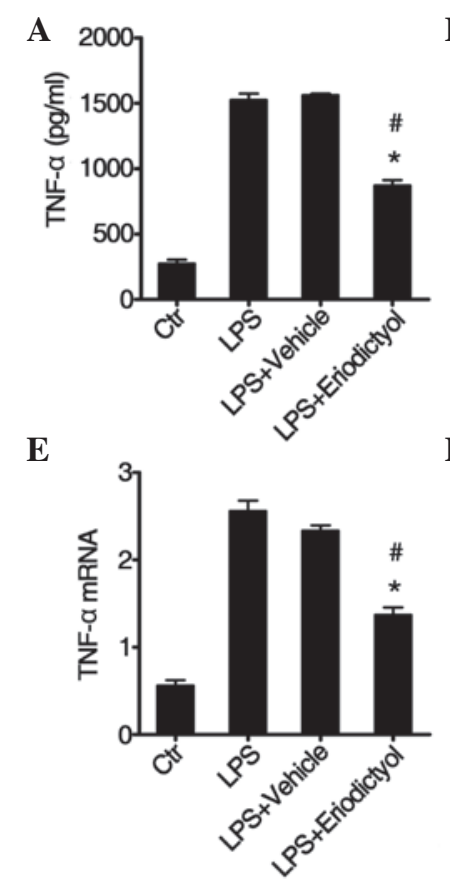

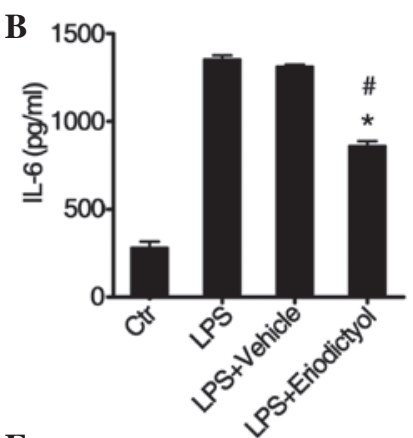

F

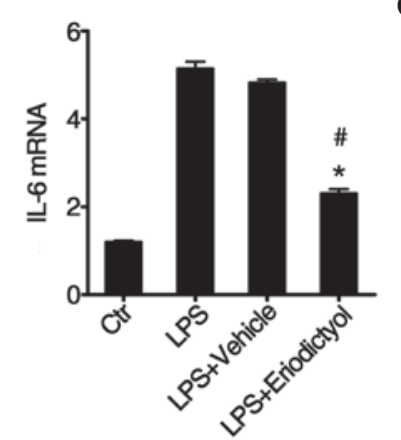

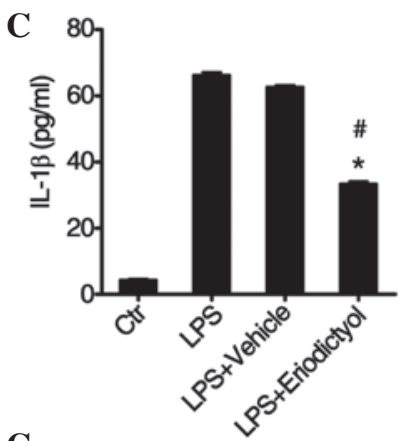

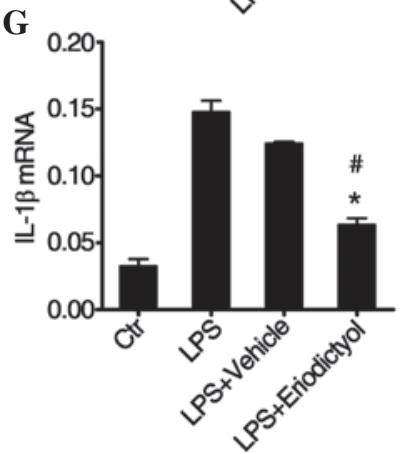

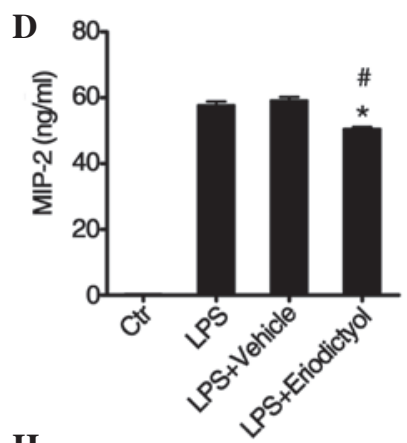

$\mathbf{H}$

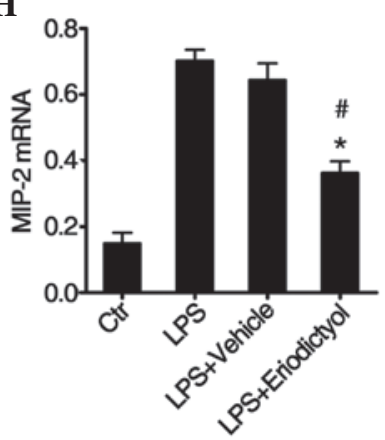

Figure 7. Effect of eriodictyol on the inflammatory cytokine expression and production in macrophages. The (A-D) ELISA and (E-H) reverse transcription-quantitative polymerase chain reaction results showed that eriodictyol inhibited the production and expression of inflammatory cytokines in macrophages. Data are presented as the mean \pm standard error $(\mathrm{n}=10)$. ${ }^{*} \mathrm{P}<0.05$ compared with LPS-induced ALI group; ${ }^{*} \mathrm{P}<0.05$ compared with the vehicle-treated ALI group. ALI, acute lung injury; Ctr, control; LPS, lipopolysaccharide; MIP-2, inflammatory protein 2; TNF- $\alpha$, tumor necrosis factor- $\alpha$; IL, interleukin. 
group, as compared with the expression in the LPS-induced or vehicle-treated ALI groups. The protein expression levels of Nrf2 and Trx1 (Fig. 6C and D) were consistent with the mRNA expression levels. These results also indicated that eriodictyol could alleviate LPS-induced ALI through its antioxidative activity.

Expression of inflammatory cytokines in macrophages is inhibited by eriodictyol pretreatment. Previous studies have demonstrated that macrophages play a critical role in the pathogenesis of lung injury, since they initiate the inflammatory response and promote neutrophil infiltration and tissue damage in the lungs (37-39). In vitro, eriodictyol has been shown to inhibit the expression of inflammatory cytokines (21); therefore, in the present study, macrophages from C57BL/6 mice were isolated and stimulated with LPS and eriodictyol or vehicle, and then the mRNA and protein levels of inflammatory cytokines produced in the macrophages were detected. It was found that the expression levels of inflammatory cytokines, such as TNF- $\alpha$ (Fig. 7A), IL-6 (Fig. 7B), IL-1 $\beta$ (Fig. 7C), and MIP-2 (Fig. 7D), were decreased in eriodictyol-treated macrophages, as compared with the LPS-treated or vehicle-treated macrophages. The mRNA expression levels of these inflammatory cytokines: TNF- $\alpha$ (Fig. 7E), IL-6 (Fig. 7F), IL-1 $\beta$ (Fig. 7G), and MIP-2 (Fig. 7H), were similar to the protein expression levels (Fig. 7A-D).

\section{Discussion}

The occurrence of ALI is strongly associated with infection with LPS-containing Gram-negative bacteria (40). In the LPS-induced ALI mouse model, the manifestations are similar to the pathological characteristics of ALI in humans (41); therefore, this model appears to be well suited for the study of potential preliminary preventive or therapeutic compounds against ALI in humans. Eriodictyol, a flavonoid found in citrus fruits, has been reported to have a potential antioxidative and anti-inflammatory activity in vitro $(34,42)$. In the present study, it was demonstrated that eriodictyol could attenuate the LPS-induced ALI in mice, thus prolonging their survival time, as well as that eriodictyol treatment could protect mice from LPS-induced ALI via inhibiting the expression of inflammatory cytokines in macrophages and activating the Nrf2 pathway to suppress oxidative injury in ALI mice.

The exudation in the early phase of LPS-induced ALI is characterized by increased levels of neutrophils, proteins and inflammatory cytokines and chemokines in the BALF and serum, depending on the severity of the disease $(43,44)$. In both systemic and local both information, edema is a typical symptom. Widespread alveolar epithelial destruction and neutrophil infiltration in the alveolar spaces, in association with high volumes of proteinaceous exudate, comprise a typical ALI lesion. MPO is an enzyme located mainly in the primary granules of the neutrophils and, thus, the activity of MPO in the parenchyma reflects the adhesion and margination of neutrophils in the lung tissues; therefore, to investigate the protective effect of eriodictyol on LPS-induced ALI, LPS-induced ALI mice were pretreated with eriodictyol, and the effect of eriodictyol pretreatment on the histology, lung tissue wet/dry ratio, protein concentration in the BALF, MPO activity and neutrophil count in the ALI mice was examined. The results of the histopathological examination showed that eriodictyol pretreatment improved the LPS-induced histological manifestations (Fig. 1A-D), including inflammatory cell infiltration, increased alveolar septum thickness, hyaline membrane formation, alveolar congestion and hemorrhage. It was also found that eriodictyol treatment decreased the lung wet/dry ratio (Fig. 1F), protein concentration (Fig. 1H) and neutrophil count (Fig. 3B) in the BALF and the MPO activity in the homogenates (Fig. 3A), compared with the LPS-induced or vehicle-treated ALI groups. These results suggest a potential therapeutic effect of eriodictyol on LPS-induced ALI.

It is believed that a sustained and uncontrolled pulmonary inflammation plays an important role in the pathogenesis of ALI $(45,46)$ and, therefore, a potential strategy to attenuate the progression of ALI could be based on suppressing the immune system-mediated inflammatory responses. It was found in the present study that eriodictyol exerts a powerful anti-inflammatory activity in the ALI mouse model. Compared with the LPS-induced or vehicle-treated ALI groups, the levels of inflammatory cytokines in the serum and BALF were significantly reduced in the ALI mice that received eriodictyol pretreatment (Fig. 2). Since the primary source of inflammatory cytokines in the ALI mice was macrophages, the macrophages were isolated and cultured with eriodictyol or vehicle, and it was found that the increase in inflammatory cytokines, such as TNF- $\alpha$, IL-6, IL-1 $\beta$ and MIP-2, which was caused by LPS stimulation, was inhibited following the administration of eriodictyol (Fig. 7). These data suggested that eriodictyol reduced LPS-induced ALI by inhibiting the expression of inflammatory cytokines in macrophages.

Excessive oxidative injury is another factor involved in the pathogenesis of ALI $(47,48)$. A previous study indicated that eriodictyol is capable of exhibiting antioxidative activity in vitro (34). In the present study it was observed that eriodictyol could enhance the activation of the Nrf2 pathway and increase the expression of antioxidant response element-regulated genes, such as Trx1, in the lung tissues from LPS-induced ALI mice. These results were supported by a study on the effects of eriodictyol in ARPE-19 cells in vitro (34).

In conclusion, the present results demonstrated that pretreatment with eriodictyol significantly attenuated pulmonary inflammation and lung injury in mice with LPS-induced ALI, and that the protective effect of eriodictyol could be, at least partly, attributed to its abilities to alleviate the excessive oxidative injury and inhibit the production of inflammatory cytokines, including TNF- $\alpha$, IL-6, IL-1 $\beta$ and MIP-2, in macrophages. In addition, the protective effect of eriodictyol in ALI may be associated with the suppression of NF- $\mathrm{NB}$ signaling and the activation of the Nrf2 pathway, which subsequently causes a marked reduction in inflammatory responses and oxidative injury in lung tissue.

\section{Acknowledgements}

This study was partly supported by a grant from the Beijing Municipal Commission of Education (grant no. M200810025006) and a grant from the Beijing Natural Science Foundation (grant no. 7112039). 


\section{References}

1. Luh SP and Chiang CH: Acute lung injury/acute respiratory distress syndrome (ALI/ARDS): The mechanism, present strategies and future perspectives of therapies. J Zhejiang Univ Sci B 8: 60-69, 2007.

2. Rubenfeld GD: Epidemiology of acute lung injury. Crit Care Med 31 (Suppl): S276-S284, 2003.

3. Zhang X, Song K, Xiong H, Li H, Chu X and Deng X: Protective effect of florfenicol on acute lung injury induced by lipopolysaccharide in mice. Int Immunopharmacol 9: 1525-1529, 2009

4. Ferrero-Miliani L, Nielsen OH, Andersen PS and Girardin SE: Chronic inflammation: Importance of NOD2 and NALP3 in interleukin-1beta generation. Clin Exp Immunol 147: 227-235, 2007.

5. Aderem A and Ulevitch RJ: Toll-like receptors in the induction of the innate immune response. Nature 406: 782-787, 2000.

6. Hsu H, Shu HB, Pan MG and Goeddel DV: TRADD-TRAF2 and TRADD-FADD interactions define two distinct TNF receptor 1 signal transduction pathways. Cell 84: 299-308, 1996.

7. Karin $\mathrm{M}$ and Lin A: NF-kappaB at the crossroads of life and death. Nat Immunol 3: 221-227, 2002

8. Liu J, Minemoto Y and Lin A: c-Jun N-terminal protein kinase 1 (JNK1), but not JNK2, is essential for tumor necrosis factor alpha-induced c-Jun kinase activation and apoptosis. Mol Cell Biol 24: 10844-10856, 2004.

9. Liu J and Lin A: Wiring the cell signaling circuitry by the NF-kappa B and JNK1 crosstalk and its applications in human diseases. Oncogene 26: 3267-3278, 2007.

10. Tang G, Minemoto Y, Dibling B, et al: Inhibition of JNK activation through NF-kappaB target genes. Nature 414: 313-317, 2001.

11. Aldridge AJ: Role of the neutrophil in septic shock and the adult respiratory distress syndrome. Eur J Surg 168: 204-214, 2002.

12. Martin TR: Cytokines and the acute respiratory distress syndrome (ARDS): A question of balance. Nat Med 3: 272-273, 1997.

13. Calkins MJ, Johnson DA, Townsend JA, et al: The Nrf2/ARE pathway as a potential therapeutic target in neurodegenerative disease. Antioxid Redox Signal 11: 497-508, 2009.

14. Touyz RM: Reactive oxygen species, vascular oxidative stress and redox signaling in hypertension: What is the clinical significance? Hypertension 44: 248-252, 2004.

15. Van Eeden S, Leipsic J, Paul Man SF and Sin DD: The relationship between lung inflammation and cardiovascular disease. Am J Respir Crit Care Med 186: 11-16, 2012.

16. Cachofeiro V, Goicochea M, de Vinuesa SG, Oubiña P, Lahera V and Luño J: Oxidative stress and inflammation, a link between chronic kidney disease and cardiovascular disease. Kidney Int Suppl 74: S4-S9, 2008.

17. Pedruzzi LM, Stockler-Pinto MB, Leite M, Jr and Mafra D Nrf2-keap1 system versus NF-kappaB: The good and the evil in chronic kidney disease? Biochimie 94: 2461-2466, 2012.

18. Deramaudt TB, Dill C and Bonay M: Regulation of oxidative stress by Nrf2 in the pathophysiology of infectious diseases. Med Mal Infect 43: 100-107, 2013.

19. Ismaili H, Sosa S, Brkic D, et al: Topical anti-inflammatory activity of extracts and compounds from Thymus broussonettii. J Pharm Pharmacol 54: 1137-1140, 2002.

20. Minato K, Miyake Y, Fukumoto S, et al: Lemon flavonoid, eriocitrin, suppresses exercise-induced oxidative damage in rat liver. Life Sci 72: 1609-1616, 2003.

21. Lee JK: Anti-inflammatory effects of eriodictyol in lipopolysaccharide-stimulated raw 264.7 murine macrophages. Arch Pharm Res 34: 671-679, 2011.

22. Zhou ZH, Sun B, Lin K and Zhu LW: Prevention of rabbit acute lung injury by surfactant, inhaled nitric oxide, and pressure support ventilation. Am J Respir Crit Care Med 161: 581-588, 2000.

23. Bradley PP, Priebat DA, Christensen RD and Rothstein G: Measurement of cutaneous inflammation: Estimation of neutrophil content with an enzyme marker. J Invest Dermatol 78: 206-209, 1982

24. Numata M, Suzuki S, Miyazawa N, et al: Inhibition of inducible nitric oxide synthase prevents LPS-induced acute lung injury in dogs. J Immunol 160: 3031-3037, 1998.
25. Lee JP, Li YC, Chen HY, et al: Protective effects of luteolin against lipopolysaccharide-induced acute lung injury involves inhibition of MEK/ERK and PI3K/Akt pathways in neutrophils. Acta Pharmacol Sin 31: 831-838, 2010

26. Nosotti M, Falleni M, Palleschi A, et al: Quantitative real-time polymerase chain reaction detection of lymph node lung cancer micrometastasis using carcinoembryonic antigen marker. Chest 128: 1539-1544, 2005.

27. Manicone AM, Birkland TP, Lin M, et al: Epilysin (MMP-28) restrains early macrophage recruitment in Pseudomonas aeruginosa pneumonia. J Immunol 182: 3866-3876, 2009.

28. Kabir K, Gelinas JP, Chen M, et al: Characterization of a murine model of endotoxin-induced acute lung injury. Shock 17: 300-303, 2002

29. Abraham E: Neutrophils and acute lung injury. Crit Care Med 31 (4 Suppl): S195-S199, 2003.

30. Grommes J and Soehnlein O: Contribution of neutrophils to acute lung injury. Mol Med 17: 293-307, 2011.

31. Cheng PY, Lee YM, Wu YS, Chang TW, Jin JS and Yen MH: Protective effect of baicalein against endotoxic shock in rats in vivo and in vitro. Biochem Pharmacol 73: 793-804, 2007.

32. Baldwin SR, Simon RH, Grum CM, Ketai LH, Boxer LA and Devall LJ: Oxidant activity in expired breath of patients with adult respiratory distress syndrome. Lancet 1: 11-14, 1986

33. Kietzmann D, Kahl R, Müller M, Burchardi H and Kettler D: Hydrogen peroxide in expired breath condensate of patients with acute respiratory failure and with ARDS. Intensive Care Med 19: 78-81, 1993.

34. Johnson J, Maher P and Hanneken A: The flavonoid, eriodictyol, induces long-term protection in ARPE-19 cells through its effects on Nrf2 activation and phase 2 gene expression. Invest Ophthalmol Vis Sci 50: 2398-2406, 2009.

35. Wang B, Zhu X, Kim Y, et al: Histone deacetylase inhibition activates transcription factor $\mathrm{Nrf} 2$ and protects against cerebral ischemic damage. Free Radic Biol Med 52: 928-936, 2012.

36. Furukawa M, Tanaka R, Chuang VT, et al: Human serum albumin-thioredoxin fusion protein with long blood retention property is effective in suppressing lung injury. J Control Release 154: 189-195, 2011

37. Frank JA, Wray CM, McAuley DF, Schwendener R and Matthay MA: Alveolar macrophages contribute to alveolar barrier dysfunction in ventilator-induced lung injury. Am J Physiol Lung Cell Mol Physiol 291: L1191-L1198, 2006.

38. Eyal FG, Hamm CR and Parker JC: Reduction in alveolar macrophages attenuates acute ventilator induced lung injury in rats. Intensive Care Med 33: 1212-1218, 2007.

39. Johnston LK, Rims CR, Gill SE, McGuire JK and Manicone AM: Pulmonary macrophage subpopulations in the induction and resolution of acute lung injury. Am J Respir Cell Mol Biol 47: 417-426, 2012.

40. Matthay MA, Ware LB and Zimmerman GA: The acute respiratory distress syndrome. J Clin Invest 122: 2731-2740, 2012.

41. Rojas M, Woods CR, Mora AL, Xu J and Brigham KL: Endotoxin-induced lung injury in mice: Structural, functional and biochemical responses. Am J Physiol Lung Cell Mol Physiol 288: L333-L341, 2005.

42. Wu Y, Singer M, Thouron F, Alaoui-El-Azher M and Touqui L: Effect of surfactant on pulmonary expression of type IIA PLA(2) in an animal model of acute lung injury. Am J Physiol Lung Cell Mol Physiol 282: L743-750, 2002.

43. Jerala R: Structural biology of the LPS recognition. Int J Med Microbiol 297: 353-363, 2007.

44. Lu YC, Yeh WC and Ohashi PS: LPS/TLR4 signal transduction pathway. Cytokine 42: 145-151, 2008.

45. Goodman RB, Pugin J, Lee JS and Matthay MA Cytokine-mediated inflammation in acute lung injury. Cytokine Growth Factor Rev 14: 523-535, 2003

46. Manicone AM: Role of the pulmonary epithelium and inflammatory signals in acute lung injury. Expert Rev Clin Immunol 5: 63-75, 2009.

47. Ward PA: Oxidative stress: Acute and progressive lung injury. Ann NY Acad Sci 1203: 53-59, 2010.

48. Imai Y, Kuba K, Neely GG, et al: Identification of oxidative stress and Toll-like receptor 4 signaling as a key pathway of acute lung injury. Cell 133: 235-249, 2008. 